\title{
CHROMOSOMAL MICRODISSECTION BY LASER
}

\author{
A Cytochemical and Functional Analysis \\ M. W. BERNS ${ }^{1}$ and A. D. FLOYD ${ }^{2}$ \\ ${ }^{1}$ Department of Zoology and ${ }^{2}$ Department of Anatomy, University of Michigan, \\ Ann Arbor, Mich. 48104, USA
}

\begin{abstract}
SUMMARY
Chromosomes of salamander lung epithelium were irradiated in vitro using an argon laser microbeam. Functional analysis was performed by testing the ability to inactivate the nucleolar organizer. Cytochemical analysis was used to detect changes in either the chromosumal DNA or protein. Results indicated that by using low laser powers in conjunction with the dye acridine orange it is possible to functionally and structurally alter the DNA without affecting the histone protein, and by using high laser powers without a photosensitizing dye, it is possible to alter histone protein without affecting the DNA or non-histone protein.
\end{abstract}

It is possible to produce selective Feulgennegative microlesions $(0.5-1 \mu \mathrm{m}$ in diameter $)$ on mitotic chromosomes [1-4]. It has also been demonstrated that specific functional loss can be associated with these lesions [5]. Irradiation of the nucleolar organizer regions (secondary constrictions) of salamander chromosomes results in the inability of those sites to contribute to nucleolar formation (i.e., a nucleolus cannot be produced). All of these studies have been conducted using a low power, pulsed argon laser (1 watt, peakpower), in conjunction with selective sensitization of the chromosomes to the laser light by pretreatment with acridine orange (AO).

Recently it has been possible to irradiate chromosomes with argon laser powers of 18-35 W. Irradiation with these high energy densities results in the production of microlesions without the addition of $\mathrm{AO}$ as a photosensitizing agent [6]. Through the phase microscope these lesions appear similar to the dye-sensitized lesions. However, preliminary cytochemical evidence has indicated that the nature of the lesions in both situations is markedly different. It has been suggested that the AO-sensitized alterations are changes in the DNA component of the chromosome, and the non-dye sensitized lesions represent protein changes [6]. If it is possible to delete selectively either protcin or DNA from desired chromosome regions of living cells, considerable insight into the relationship of these components to basic chromosome structure and function might be gained. Consequently, we undertook to define cytochemically and functionally the nature of these chromosome lesions.

\section{MATERIALS AND METHODS}

The tissue culture procedures are identical to those described in previous publications [1-6]. Lung tissue from the salamander Taricha granulosa is established as primary explants in Rose multipurpose culture 
chambers. Culture medium is a standard Eagle MEM, fortified with antibiotics and $10 \%$ fetal calf serum. One to three weeks following setup epithelial outgrowth with many mitotic cells are evident.

Microbeam irradiation is performed using a pulsed argon laser (major wavelengths are $488 \mathrm{~nm}$ and 514 $\mathrm{nm}$ ) with a peak output of 35 watts/pulse. Pulse duration is $50 \mu \mathrm{sec}$. The laser is complexed with a Zeiss photomicroscope and closed circuit television unit so that the specimen can be viewed continually during irradiation. A dichroic interference filter is used to direct the laser beam down into the microscope while at the same time permitting the image of the specimen to be directed to the television monitor. The desired chromosome is moved under a cross hair on the television monitor, and the laser is fired. The amount of laser energy entering the microscope objective is controlled in several ways: (1) by varying the input voltage to the laser tube; (2) utilizing calibrated neutral density filters between the laser head and the microscope system; (3) using different focal length lenses between the laser and the microscope system. The actual amount of energy contained within the focal spot of the microscope is measured with a calibrated S-5 vacuum photodiode. Maximum measured energy in the focal spot can be as high as 1050 microjoules $(\mu \mathrm{J})$. In the experiments presented in this manuscript the maximum energy density in the focal spot is about $300 \mu \mathrm{J}$.

The actual diameter of the focused spot is around 1 $\mu \mathrm{m}$, but, because the laser is utilized in single mode configuration, the energy density across the focused spot is a gaussian distribution. Thus, the diameter of the effective energy (the energy density above the lesion threshold of the target) may be as small as $0.25 \mu \mathrm{m}$. Lesions of this diameter have been produced routinely.

Chromosome microirradiation was performed on cells that were pretreated with acridine orange $(\mathrm{AO})$, and on cells that were not treated with the dye. Dye-treated cells were subjected to $\mathrm{AO}, 0.1 \mu \mathrm{g} / \mathrm{ml}$ of culture medium for $5 \mathrm{~min}$, washed twice with sterile Hanks balanced salt solution, and fresh culture medium was added. Irradiation occurred 5-30 min following the washes. Non-dye treated cells were washed with balanced salt solution followed by addition of fresh culture medium. Irradiation followed the washes.

Photographs were taken before and after irradiation. After the post-irradiation photographs, the cells were either observed in vitro for several hours, or fixed and stained cytochemically.

In the non-cytochemical series of experiments, the secondary constriction regions of anaphase chromosomes were irradiated as previously described [5]. Secondary constriction regions of both AO-treated and non-AO-treated cells were irradiated, and subsequent nucleolar formation noted.

Cytochemical analysis also was performed on both dye- and non-dye-treated cells. Several different cytochemical procedures were undertaken to determine whether the laser lesions represented changes in specific chromosomal components (i.e., DNA and/or protein). DNA-purines was demonstrated with a standardized Feulgen procedure [7] and DNAphosphate by the gallocyanin-chrome alum technique
[8]. The Alfert \& Geschwind alkaline fast green procedure for basic proteins (histones) was employed as cited by Deitch [9]. General proteins (proteins not masked by combination with DNA) were visualized with naphthol yellow $S$ [9].

\section{RESULTS}

\section{Secondary constriction irradiation}

Irradiation of secondary constriction regions following $\mathrm{AO}$ treatment resulted in the inability of the irradiated region to organize a nucleolus. A nucleolus was organized by the same chromosome region in the daughter cell. The daughter cell was irradiated in a non-secondary constriction region. This is in agreer ant with earlier reports [5]. Irradiation of secondary constriction regions in cells that had not been treated with AO, resulted in a definite lesion to the chromosome, but did not affect the ability of that region to organize a nucleolus. These results are illustrated in fig. 1. Note the definite "phase paling" of the irradiated region. This experiment was repeated three times with the same results. In all three experiments the laser was fired four times at the secondary constriction resulting in a cumulative imposed energy of $1200 \mu \mathrm{J}$.

\section{Cytochemical analysis}

As in previous studies [2, 3] the lesion areas of AO-treated cells were negative staining when analysed by the Feulgen reaction. However, when the chromosomes from the AO-treated cells were stained for basic protein (histone) with alkaline fast green, no difference in staining could be detected between the lesion area and the unirradiated area (fig. $2 a, b . c$ ). Energy densities in these experiments was not above $50 \mu \mathrm{J} /$ pulse. A maximum of three pulses $(150 \mu \mathrm{J})$ was used.

Lesions produced on non AO-treated chromosomes stained differently than the lesions 

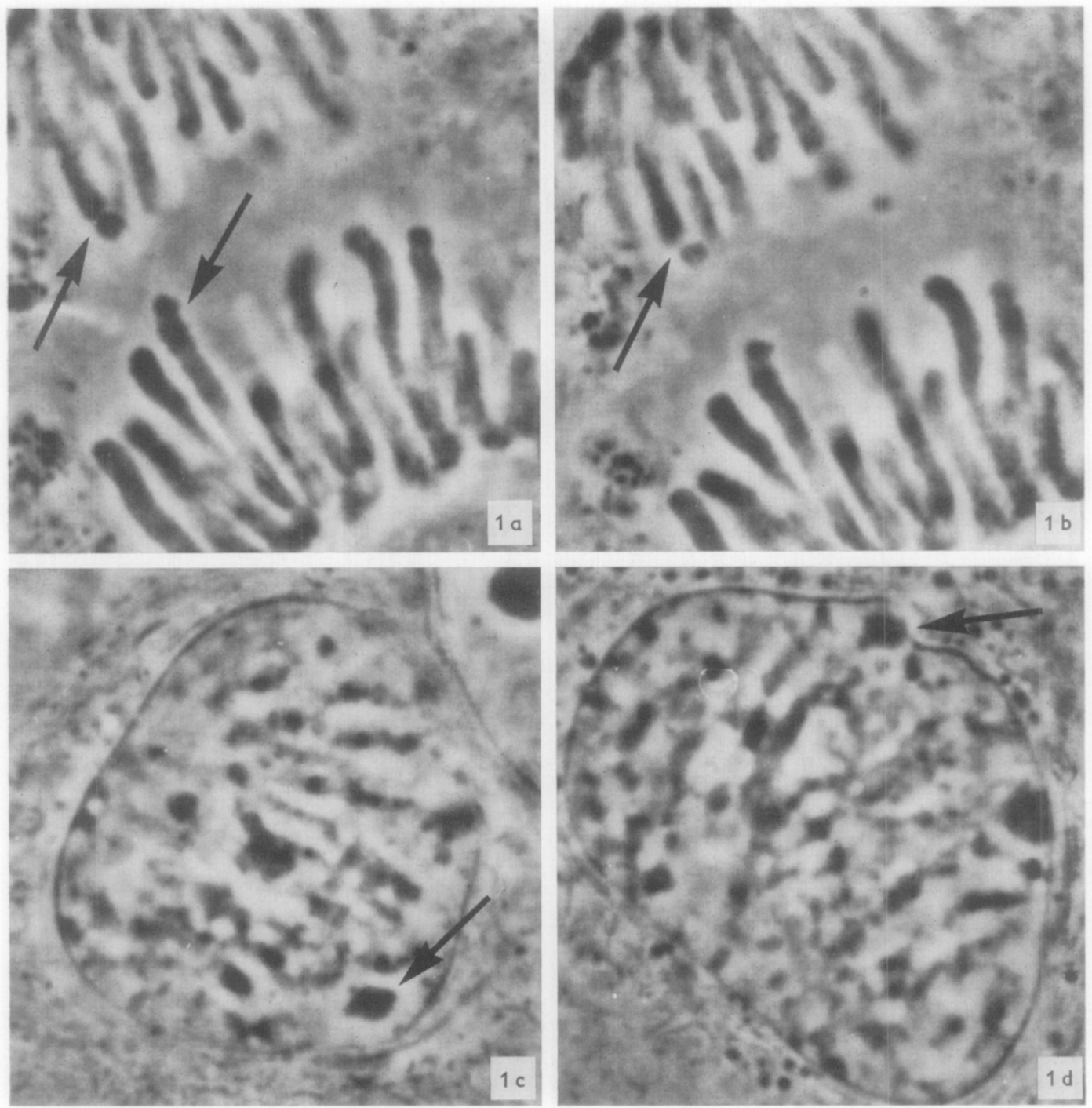

Fig. 1. Irradiation of secondary constriction $\mathrm{n}$ a non-AO-treated cell. (a) Pre-irradiation. Top arrow shows constriction to be irradiated; lower arrow is control constriction. (b) Post-irradiation, laser fired 4 times, cumulative cnergy $1200 \mu \mathrm{J}$. Note the paled lesion area. (c) Post-mitotic nucleus coniaining irradiated secondary constriction. Arrow indicates nucleolus formed in association with constriction. $(d)$ Nucleus containing control secondary constriction. Arrow indicates nucleolus produced by the control region. $\times 3000$ (all cells).

of the AO-treated chromosomes. DNAFeulgen staining revealed no difference between the irradiated and unirradiated areas (fig. $2 d, e, f$ ). However, alkaline fast green staining for histone protein was negative in the lesion area (fig. $3 a, b$ ). Staining for nonhistone protein with napthol yellow indicated no change in the lesion area (fig. $3 c, d$ ). In addition, gallocyanine-chrome alum staining for DNA also revealed little or no change in the lesion area. In all of the above experiments, total energy densities were 1200 $1500 \mu \mathrm{J}$ ( 4 to 5 pulses per experiment). Each experiment was repeated at least three times. 

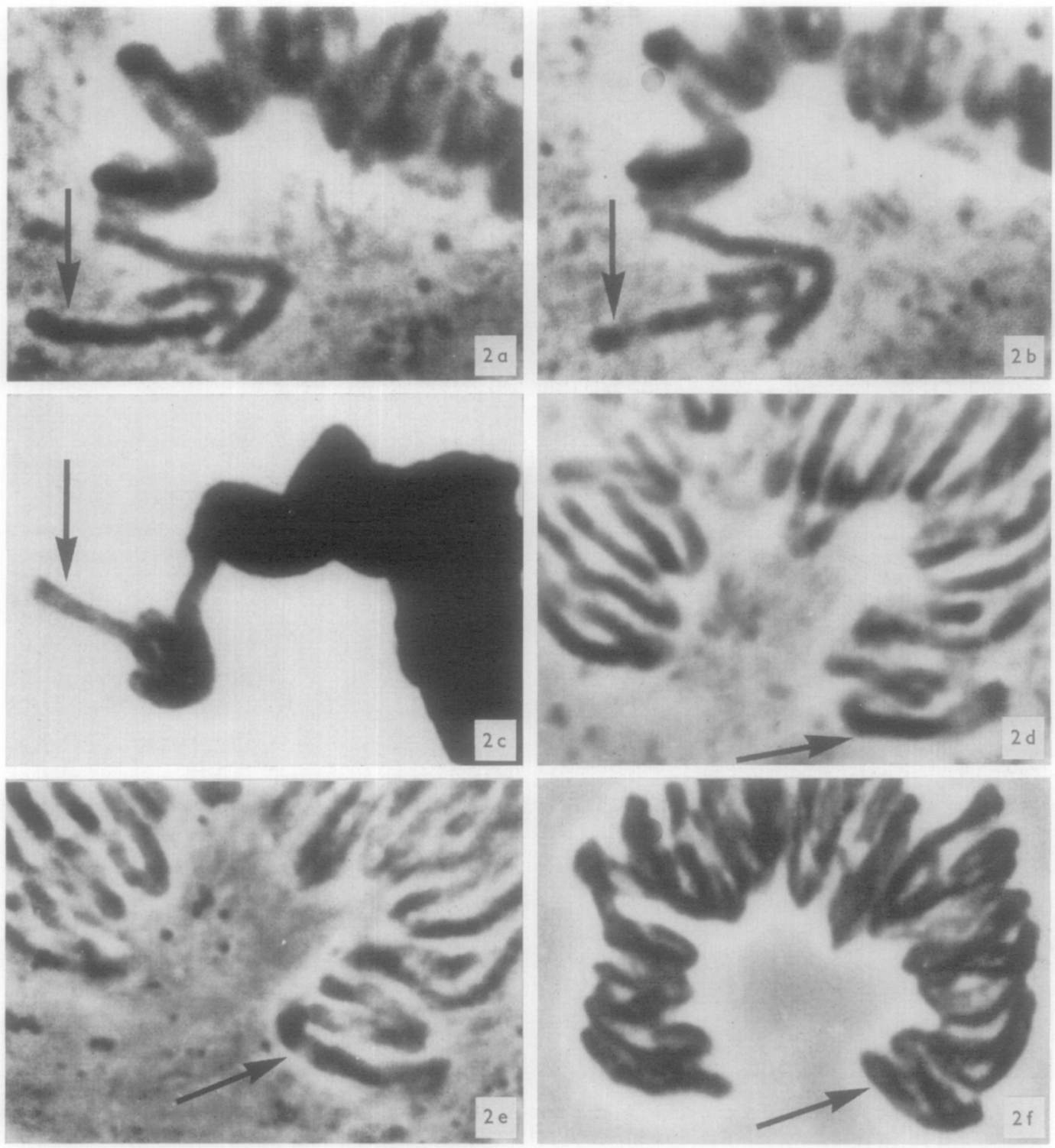

Fig. 2. (a-c) AO-treated chromosomes following laser irradiation and subsequent histone staining with alkaline fast green. (a) Pre-irradiation chromosome arm (arrow); (b) post-irradiation lesion (arrow) prior to fixation; (c) same chromosome region (arrow) following staining procedure. $\times 3000$. Total imposed laser energy, 150 $\mu \mathrm{J} .(d-f)$ Non-AO-treated chromosomes following laser irradiation and subsequent staining by the Feulgen reaction for DNA. (d) Pre-irradiation; (e) post-irradiation, 4 pulses, cumulative energy $1200 \mu \mathrm{J}$; $(f)$ same chromosome region following fixation and staining. $\times 3000$.

\section{DISCUSSION}

It would appear that by varying appropriate parameters, either chromosomal DNA or DNA associated protein can be selectively affected. The lesions of AO-treated chromosomes seem to involve changes to the DNA rather than the basic protein. This is suggested by the negative Feulgen staining and the positive alkaline fast green staining. 

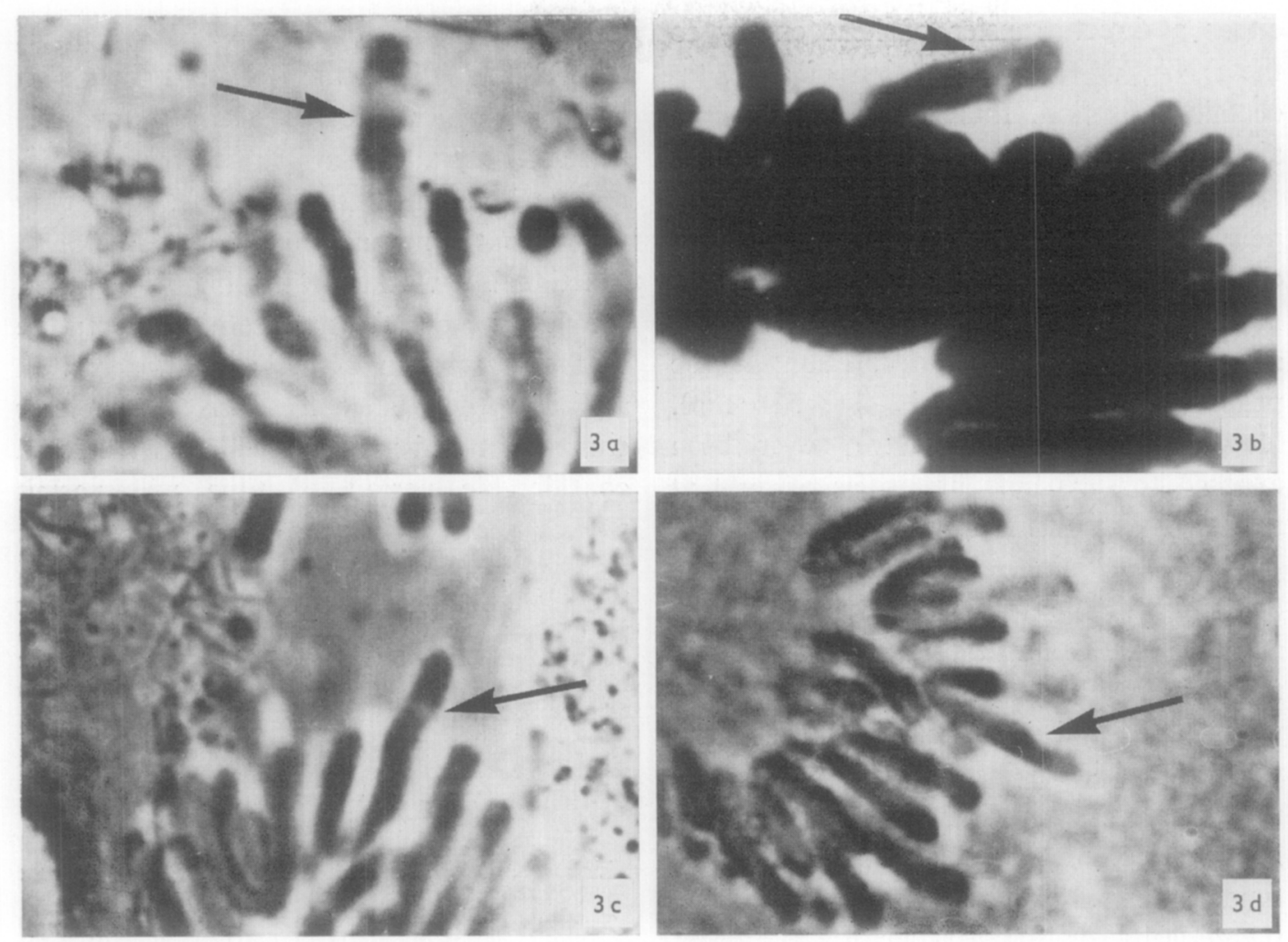

Fig. 3. Non-AO-treated chromosomes. ( $a-b)$ Irradiated and stained for histone. (a) Post-laser living; (b) fixed and stained. $(c, d)$ Stained for general protein (non-DNA associated). (c) Post-laser, pre-fixation; $(d)$ stained with naphthol yellow. Arrows indicate lesion sites. $\times 3000$. Total imposed laser energy, $1200-1500 \mu \mathrm{J}$.

The lesion produced with the high laser powers, without the use of $\mathrm{AO}$ as a photosensitizing agent, appear to be selective for basic protein. This is suggested by the negative alkaline fast green staining (for basic protein), positive Feulgen staining (for DNA purine bases), positive gallocyanine staining (for DNA phosphate), and positive naphthol yellow (for non-histone protein). The ability to produce a definite lesion in the secondary constriction region of the chromosome without affecting the ability of that region to organize a nucleolus would also suggest a change to the protein component rather than the DNA. This result would also indicate that the altered protein component does not play a critical functional role in the organi- zation of nucleolus. It has been suggested by several authors $[10,11]$ that the nucleolar organizing secondary constrictions contain the ribosomal poly-cistrons that are directly involved in nucleolar synthesis. One would expect that disruption of this segment of the DNA would result in an altered nucleolar synthesis. This is the result observed when the secondary constriction regions of $\mathrm{AO}$ treated chromosomes are irradiated.

The mechanism of lesion production can only be speculated about at this time. In the case of the AO-treated chromosomes, it seems likely that the DNA is being altered because the AO intercalates directly between the base pairs of the DNA molecule. Absorption of energy by the AO molecule could result in 
either a direct transfer of heat energy to the DNA, or the precipitation of a photochemical reaction. Either mechanism could alter the DNA appreciably.

The alteration to the basic protein component in the non-acridine orange treated chromosomes is less easy to explain. There is no indication in the literature that chromosomal protein has any absorption at the primary wavelengths used (488, $514 \mathrm{~nm}$ ). However, chromosomes have never been exposed to such high intensities of light at these wavelengths. The possibility of a two photon absorption effect [13], cannot be overlooked. The result would be twice the amount of quanta, therefore, exactly half the wavelength (244 $\mathrm{nm}$ for the $488 \mathrm{~nm}$ wavelength, and $257 \mathrm{~nm}$, for the $514 \mathrm{~nm}$ wavelength). With increasing photon densities, the probability of two photons striking a target molecule at exactly the same time increases. It would, therefore, be possible theoretically for this to occur in a microspot with a high photon density. However, one would expect greater damage to DNA than protein, since the two photon wavelengths would be 244 and $257 \mathrm{~nm}$.

Another possible explanation of the nonAO lesions is that a sensitizing agent other than AO is present. Though this seems unlikely, it cannot be entirely ruled out. It might also be possible that the artificial culture environment has changed the natural absorbance of the chromosomes.

Whatever the explanation for the effect, it is possible to selectively alter either DNA or basic protein by simply varying the energy in the focused spot, and the presence of a vital dye, acridine orange. With the dye present, and using relatively low laser output
(50 $\mu \mathrm{J} /$ pulse), it is possible to selectively affect the DNA. Without the dye, and with high energy densities ( $300 \mu \mathrm{J} /$ pulse) it is possible to selectively alter the histone protein apparently without affecting the DNA. The ability to alter one component both structurally and functionally without affecting the other, should raise questions concerning the nature of the association of these components. In addition, the ability to dissect out either component and maintain cell survival for a considerable period of time will permit studies of a functional nature.

We would like to thank Wanny $K$. Cheng for her aid in setting up the tissue cultures.

This research was financed by grants from the National Science Foundation, GB 24457, American Cancer Society institutional grant IN40K.

\section{REFERENCES}

1. Berns, M W, Olson, R S \& Rounds, D E, Nature 221 (1969) 74.

2. Berns, M W, Rounds, D E \& Olson, R S, Exptl cell res 56 (1969) 292.

3. Berns, M W \& Rounds, D E, Ann NY acad sci 168 (1970) 550.

4. - Sci Am 222 (1970) 98.

5. Berns, M W, Ohnuki, Y, Rounds, D E \& Olson, S, Exptl cell res 60 (1970) 133.

6. Berns, M W, Cheng, W K, Floyd, A D \& Ohnuki, Y, Science 171 (1971) 903.

7. Leuchtenberger, $C$, General cytochemical methods (ed J F Danielli) vol. 1, p. 219. Academic Press, New York (1958).

8. Sandritter, W, Kiefer, G \& Rick, W, Introduction to quantitative cytochemistry (ed G L Wied) p. 295. Academic Press, New York (1966).

9. Deitch, A D, Introduction to cytochemistry (ed G L Wied) p. 327. Academic Press, New York (1966).

10. Hsu, T C, Brinkley, B R \& Arrighi, F E, Chromosoma (Berlin) 23 (1967) 137.

11. Brown, D D \& Dawid, I B, Science 160 (1968) 272.

12. Rounds, D E, Olson, R S \& Johnson, F M, NEREM Rec (1966) 158.

13. Kleinman, D A, Phys rev 125 (1962) 87.

Received March 3, 1971 